\title{
The Role of Intermolecular Interactions in the Electro-Optical Kerr Effect in Liquid Alkanes
}

\author{
O.V. Prezhdo ${ }^{a}$, L. SwiteK $^{b}$, V.V. Zubkova ${ }^{b}$ \\ AND V.V. PREZHDO ${ }^{b}$ \\ ${ }^{a}$ Department of Chemistry, University of Washington, Seattle, WA 98 115, USA \\ ${ }^{b}$ Institute of Chemistry, Jan Kochanowski University \\ Chęcińska 5, 25-020 Kielce, Poland
}

(Received February 21, 2005)

\begin{abstract}
The electro-optical Kerr effect of $n$-alkanes from $\mathrm{C}_{5}$ to $\mathrm{C}_{16}$ was investigated in gas phase, liquid phase, and solution. The values of the Kerr constants in gas phase are noticeably distinguishable from those in liquids, where the molecules interact by the London dispersion forces. Both the energy of the dispersion interaction and the difference in the Kerr constants in liquid and gas phase grow with molecular size, indicating the key influence of the interaction on the magnitude of the Kerr effect. Several orientation and molecular-statistical theories of electro-optical Kerr effect were applied to model the change in electro-optical Kerr effect due to the dispersion force. Most theories show significant divergence compared to the experimental data. It is argued that the decisive contribution to electro-optical Kerr effect in liquids and the deviations between the experiment and theory arise due to local liquid structures that collectively orient in the external electric field.
\end{abstract}

PACS numbers: 31.70.Dk, 78.20.Fm

\section{Introduction}

Starting from the breakthrough experiments by Kerr, electrical birefringence has become a subject of active experimental and theoretical research. The continuous interest in the electro-optical Kerr effect (EOKE) stems from its ability to provide valuable information on optical and electric properties of isolated atoms and molecules, as well as their geometric structure. Intermolecular interactions (IMI) in condensed phase [1-4] have a particularly strong influence on EOKE [5-7] making it indispensable for studies of solutions and pure liquids.

The orientation theory developed by Langevin and Born (LB) for the description of EOKE [8] is supported by the experimental results for gases and vapors. 
The theory computes the energy of interaction of a polar or anisotropically polarized molecule with the electric field. The values of the Kerr constants of pure liquids calculated using the LB formula $\left(B_{\mathrm{LB}}\right)$ are significantly higher than the experimental values $\left(B_{\exp }\right)$. The ratio $B_{\mathrm{BL}} / B_{\exp }$ is on the order of $1.5 \div 2.0[3,9-11]$ and in case of polar liquids grows with increasing molecular dipole. The success of the LB theory of EOKE for gases and its breakdown for liquids may be attributed to the internal Lorentz field that provides a good description of the response of a system of weakly-interacting gas molecules, but does not take into account the significant role of IMI in liquids. The advancements of the EOKE theory for liquids have taken place in the directions of: (1) improvement of the internal field model of liquid under the assumption that the electrical and optical properties of molecules are unchanged from gas to liquid [12]; (2) account of the correlation in molecular orientations and the resulting change in the molecular parameters [2]; (3) account of the electric field effects that are not included in the LB theory on birefringence and that contribute to the polarizability anisotropy of aggregates [13].

An advanced account for the internal field $[12,14]$ improves the ratio of the theoretical and experimental values of the Kerr constants of non-polar liquids to $B_{\text {theor }} / B_{\exp }=1 \pm 0.5$, a small average error, but still large deviations for individual compounds. The correction to the internal field is too large for many polar compounds, such as pyridine, dichlorobenzenes, ketones, etc., the result is small theoretical values $B_{\text {theor }}<B_{\exp }$. The mean-field theory of binary mixtures [15] requires a further correction in terms of the interaction parameter $\Lambda_{\mathrm{mAB}}$ [16].

The structure and dynamics of organic liquids has been the subject of intense investigation for many years. Neutron and X-ray diffraction studies of alkanes, for example, show that short-range arrangement of molecules in a liquid is similar to that in a solid $[17,18]$. The studies of the liquid structure suggest that the short-range order should be taken into account in the description of EOKE in liquids.

The paper is organized in the following way. The next section reviews the available theories of EOKE of liquids. The procedure for the measurement of the Kerr effect in an alternating electric field is described in the "experimental procedure" section. The results of the measurements in a series of $n$-alkanes are presented in the "results and discussion" section, which compares the EOKE theories and interprets the experimental data from the theoretical viewpoint. The sources of the deviations observed between the theories and experiment are analyzed in detail. The paper ends with a "conclusions" section.

\section{Modification of the orientation and molecular statistical theories of Kerr effect in condensed media}

The modifications of the internal field in the orientation theory of EOKE can be classified into two groups: (1) methods that preserve the Lorentz functional form for the internal field contribution to the Kerr constant [12, 14, 19, 20]; and 
(2) methods that replace the Lorentz function by a more complex model of the internal field [14].

Developing an approach of the first type, Stuart [19] derived the following equation for the Kerr constant $B$ :

$$
B_{\mathrm{S}}=3 \pi\left(\Theta_{1}+\Theta_{2}\right) \frac{N}{n \lambda}\left(\frac{\varepsilon+2}{3}\right)^{2}\left(\frac{n^{2}+2}{3}\right)^{2} .
$$

Here $N$ is the number of molecules in a unit volume, $n$ is refractive index, $\lambda$ is the wavelength of the analyzing light, $\varepsilon$ is static electrical permittivity, $\Theta_{1}$ and $\Theta_{2}$ are the anisotropic and dipole contributions to the Kerr constant defined by

$$
\begin{aligned}
& \Theta_{1}=(45 k T)^{-1}\left[\left(a_{1}-a_{2}\right)\left(b_{1}-b_{2}\right)+\left(a_{2}-a_{3}\right)\left(b_{2}-b_{3}\right)+\left(a_{3}-a_{1}\right)\left(b_{3}-b_{1}\right)\right], \\
& \Theta_{2}=\left(45 k^{2} T^{2}\right)^{-1}\left[\mu_{1}^{2}\left(2 b_{1}-b_{2}-b_{3}\right)+\mu_{2}^{2}\left(2 b_{2}-b_{3}-b_{1}\right)+\mu_{3}^{2}\left(2 b_{3}-b_{1}-b_{2}\right)\right],
\end{aligned}
$$

where $k$ is the Boltzmann constant, $T$ is temperature in $\mathrm{K}, a_{i}$ is static polarizability, $b_{i}$ is optical polarizability, $\mu_{i}$ is projection of the dipole moment on the $i$-axis. For non-polar liquids $(\mu=0)$ Eq. (1) transforms into

$$
B_{\mathrm{S}}=\frac{2 \pi}{15 k T} \gamma^{2} \frac{N}{n \lambda}\left(\frac{\varepsilon+2}{3}\right)^{2}\left(\frac{n^{2}+2}{3}\right)^{2},
$$

where $\gamma^{2}=\left[\left(b_{1}-b_{2}\right)^{2}+\left(b_{2}-b_{3}\right)^{2}+\left(b_{3}-b_{1}\right)^{2}\right] / 2$ is molecular polarizability anisotropy.

Considering an isotropic field acting in an anisotropic medium, Vuks $[12,14]$ derived the following expression for the Kerr constant:

$$
B_{\mathrm{V}}=3 \pi\left(\Theta_{1}+\Theta_{2}\right) \frac{N}{n \lambda}\left(\frac{\varepsilon+2}{3}\right)\left(\frac{n^{2}+2}{3}\right),
$$

where the $(\varepsilon+2) / 3$ and $\left(n^{2}+2\right) / 3$ are present to the first rather than second power as in Eq. (1). For non-polar liquids Eq. (3) simplifies to

$$
B_{\mathrm{V}}=\frac{2 \pi}{15 k T} \gamma^{2} \frac{N}{n \lambda}\left(\frac{\varepsilon+2}{3}\right)\left(\frac{n^{2}+2}{3}\right),
$$

where $\gamma^{2}$ is molecular polarizability anisotropy defined in Eq. (2).

The phenomenological theory of EOKE put forward by Zamkov [21] considers the energy of a molecule in an electrical field and results in the equation

$$
B_{\mathrm{Z} 1}=3 \pi\left(\Theta_{1}+\Theta_{2}\right) \frac{N}{n \lambda}\left(\frac{\varepsilon+2}{3}\right)\left(\frac{n^{2}+2}{3}\right)^{2}
$$

that contains $\left(n^{2}+2\right) / 3$ squared as in $(1)$ and $(\varepsilon+2) / 3$ to the first power as in (3). For non-polar molecules the theory of Zamkov gives

$$
B_{\mathrm{Z} 1}=\frac{2 \pi}{15 k T} \gamma^{2} \frac{N}{n \lambda}\left(\frac{\varepsilon+2}{3}\right)\left(\frac{n^{2}+2}{3}\right)^{2} .
$$


A second expression for the Kerr constant derived by Zamkov [22] arises by consideration of the Bettcher internal field

$$
B_{\mathrm{Z} 2}=\frac{\pi N n}{3 \lambda}\left(\frac{\varepsilon+2}{3}\right)\left(\frac{n^{2}+2}{n^{2}}\right) \sum \Theta .
$$

Here, the sum $\sum \Theta=\Theta_{1}+\Theta_{2}+\Theta_{3}$ contains three rather than two contributions, with the additional $\Theta_{3}$ contribution introduced by Fought. For non-polar molecules $\sum \Theta=\Theta_{1}$.

The molar Kerr constant $\left({ }_{m} K\right)$ is defined $[14,23]$ as the birefringence induced by one mole of a compound and is a unique characteristic of the compound. The ${ }_{m} K$ values and related properties of the compounds under study are given in the molecular anisotropy handbook [24]. Other molecular characteristics relevant for the present study were taken in Ref. [25]. As explicitly defined by Briegleb in Ref. [23] the magnitude of ${ }_{m} K$ equals to the difference in the molecular refractions of light with the orientations of the electric vector perpendicular and parallel to the field, observed over $1 \mathrm{~cm}$ in the unit field.

The use of the internal Lorentz field leads to the following equation for the molar Kerr constant [2]:

$$
{ }_{m} K_{\mathrm{K}}=\frac{6 B \lambda n}{\left(n^{2}+2\right)^{2}}\left(\frac{3}{\varepsilon+2}\right)^{2} \frac{M}{d} .
$$

The expression for the molecular Kerr derived by Le Fevre and Le Fevre [26] reads

$$
{ }_{m} K_{\mathrm{L}}=\frac{6 B \lambda n}{(\varepsilon+2)^{2}\left(n^{2}+2\right)^{2}} \frac{M}{d} .
$$

Starting from Eq. (3) Vuks [14] derived the formula

$$
{ }_{m} K_{\mathrm{V}}=\frac{9 B \lambda n}{(\varepsilon+2)\left(n^{2}+2\right)} \frac{M}{d} .
$$

Briegleb [23] determined the molar Kerr constant as

$$
{ }_{m} K_{\mathrm{B}}=\frac{B \lambda n}{\left(n^{2}+2\right)^{2}(\varepsilon+2)^{2}} \frac{M}{d} .
$$

Finally, the Onsager theory [27] results in

$$
{ }_{m} K_{\mathrm{O}}=\frac{1}{(1-a f)(1-b g)}\left({ }_{m} K^{0}-\frac{2 \pi N}{9} a f \theta_{1}\right),
$$

where $f=2(\varepsilon-1) / r^{3}(2 \varepsilon+1) ; g=2\left(n^{2}-1\right) / r^{3}\left(2 n^{2}+1\right) ; r$ is the average radius of a molecule, ${ }_{m} K^{0}$ is the molecular Kerr constant of isolated molecules, $a$ and $b$ are constants. The relationship between ${ }_{m} K_{\mathrm{L}}$ and ${ }_{m} K_{\mathrm{O}}$ can be expressed only in terms of the macroscopic parameters as

$$
{ }_{m} K_{\mathrm{O}}={ }_{m} K_{\mathrm{L}}\left[\frac{\left(2 \varepsilon+n^{2}\right)(\varepsilon+2)}{3 \varepsilon\left(n^{2}+2\right)}\right]^{2} .
$$


The early theories of the Kerr effect [8] assume that molecules in a liquid are statistically independent and can orient in any direction in space. However, molecules in condensed phase interact and correlate in orientation with the nearest neighbors.

A general statistical-mechanical equation for the calculation of the Kerr constant based on the potential energy of the molecular interaction was derived by Kielich [28]. The equation takes into account the angular correlation. The application of the Kielich theory to specific cases is significantly more difficult compared to the approaches discussed above, since it requires complex numerical simulations.

The statistical-mechanical theory of Buckingham [29] considers IMI between molecules as well as the dependence of molecular polarizability on the field intensity. The theory involves complex models and parameters that are rather difficult to determine. Similarly to the Kielich theory, the Buckingham theory of the Kerr effect is much less straightforward in practice than Eqs. (1)-(13).

The contribution of molecular hyperpolarizability into the Kerr constant is estimated in Ref. [30]. An effort to construct a complete theory of EOKE in the condensed phase including the contributions due to hyperpolarizability, angular correlations, electrostriction, and Joule effect was undertaken by Proutiere [31].

A statistical-mechanical EOKE theory for non-polar liquids was developed by Ladanyi and Keyes in Refs. [32, 33]. The theory describes IMI with the KirkwoodIvon model and uses the Lorentz field. The latter is reliable for non-polar media, but is not designed for polar molecules.

An attempt to take into account the super-molecular structure of condensed matter with the purpose of improvement of the statistical EOKE theory on the basis of the approach by Kirkwood and Frelich was undertaken in Ref. [34]. The theory considers the properties of a microscopic sphere selected in a medium. The liquid inside the sphere is treated at the microscopic level, while the outside of the sphere is simulated as continuum. The average electro-optical parameters of the system are computed with explicit regard to the intermolecular correlations inside the sphere and interaction with the outside medium. Reference [35] showed that T-shape nearest-neighbor molecular correlations are characteristic of liquid aromatic compounds. These correlations were observed earlier with X-ray dispersion in liquids [36]. Although the theoretical attempt [34, 35] undertaken to account for the nearest-neighbor correlations in liquids reflects correct physics, the practical application of the derived formulas to polar and non-polar liquids resulted in errors exceeding the errors of the simpler models based on the orientation EOKE theory.

Given the status of the existing theories, it is necessary to conclude that the influence of IMI on EOKE can be most reliably estimated only based on the experimental data. In this regard, $n$-alkanes constitute one of the simplest classes of liquids where the nearest-neighbor correlations induced by IMI and their role in EOKE can be analyzed theoretically and studied experimentally. 


\section{Experimental procedure}

\subsection{Measurement of the Kerr effect in a variable electric field}

The EOKE was investigated with a modernized version of the setup described in Ref. [37]. The advantages of the setup include minimal time of impact of the electric field on the system achieved by the impulse technique [15, 38], minimal strength of the electric field in the Kerr cell, and absence of electrolysis in the cell.

The block diagram of the apparatus is given in Fig. 1. The helium-neon laser LG-55 ( $\lambda=632.8 \mathrm{~nm}$ ) (I) was used as the light source. The electronics setup consists of the system for stabilization of the laser beam intensity (II), power source (III), high-voltage meter (IV), and registration amplifier (V). The system for laser intensity stabilization (II) sends a feedback signal through the amplifier that controls the vertical deviation in oscillograph CI-15. The power supply (III) for the Kerr cell includes the sound generator G3-33 and capacity amplifier U4-27. The amplifier output goes into the high-voltage transformer NOM-6. The electric field generated by the system can be continuously controlled up to the $10 \mathrm{kV}$ voltage at frequencies ranging from 25 to $15000 \mathrm{~Hz}$.

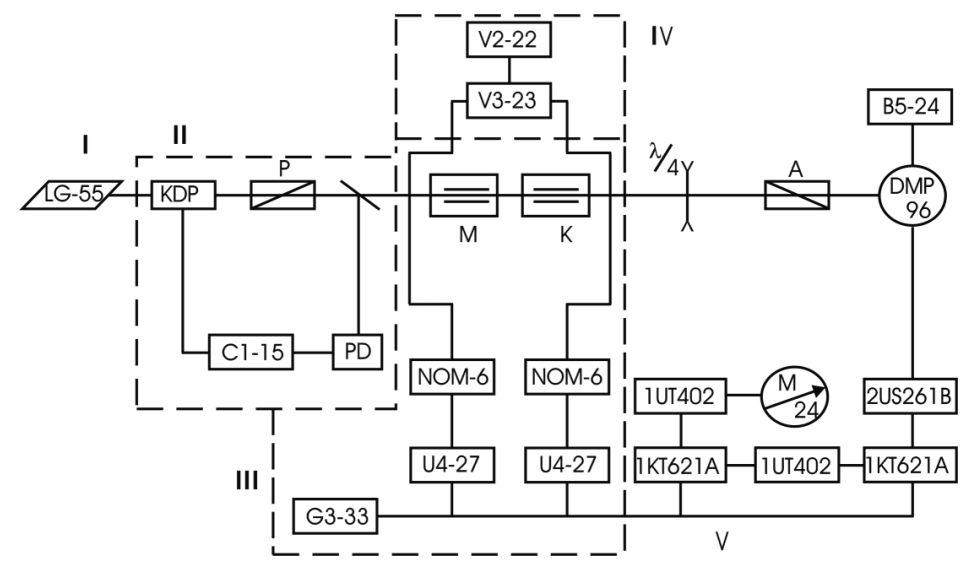

Fig. 1. The block-scheme of the apparatus for the electro-optical Kerr effect measurements. A detailed description is given in the main text.

The measuring $(\mathrm{M})$ and compensative $(\mathrm{K})$ cells of the relative measurement setup were located on the same optical axis. The high voltage inside the cells was determined with the digital voltmeter V2-22. The accuracy of the converter V3-23 was within $0.07-0.1 \%$ at frequencies from $400 \mathrm{~Hz}$ to $10 \mathrm{kHz}$ and $0.5 \%$ at frequencies from $50 \mathrm{~Hz}$ to $400 \mathrm{kHz}$, allowing for the accuracy of the cell voltage within about $0.1 \%$.

The registration amplifier was placed in the same case with the photomultiplier PMP-96. The PMP was fed with the high voltage source B5-24. The broad-band amplifier was based on the integrated microcircuit 2UC261B. The narrow- 
-band and constant-current amplifiers were made with the integrated microcircuits 1UT402. The synchronous detector was made with the integrated keys of the 1KT621A type.

The liquid layer in the quartz thermal cell was $6 \mathrm{~cm}$ thick. The titanium electrodes were $4 \mathrm{~cm}$ in length. The design of the cell allowed filling it with liquid without air access. The Frank-Ritter prisms of the $10 \times 10 \mathrm{~mm}$ size were used for the polarizer $(\mathrm{P})$ and analyzer $(\mathrm{A})$ of light. The $\lambda / 4$ plate served for transformation of the system into the linear measurement mode.

The measuring $(\mathrm{M})$ and reference $(\mathrm{K})$ cells were mounted at the $45^{\circ}$ angle to the polarizer plane and had either mutually perpendicular or mutually parallel arrangement of the electrodes, depending on the sign of birefringence in the reference and measuring cells. The sign of the birefringence arising in the first cell should be inverse to the sign of the birefringence generated in the second cell. The compensation between the two cells resulting in the absence of total birefringence and disappearance of the variable component of the photomultiplier current was controlled by the voltage on the electrodes. The compensation condition is

$$
\frac{B_{\mathrm{st}}}{B_{x}}=\text { const }_{1} \frac{E_{x}^{2}}{E_{\mathrm{st}}^{2}},
$$

where $B_{\mathrm{st}}$ is the Kerr constant of the reference substance, $B_{x}$ is the Kerr constant of the substance under study, const $_{1}$ is a cell constant determined by calibration, when both cells are filled with the same substance, $E_{\text {st }}$ and $E_{x}$ are the intensities of the electric fields in the corresponding cells.

A uniform electric field between electrodes in a Kerr cell legitimizes replacement of the electric field in Eq. (14) with voltage

$$
\frac{B_{\mathrm{st}}}{B_{x}}=\text { const }_{2} \frac{U_{x}^{2}}{U_{\mathrm{st}}^{2}},
$$

where $U_{\text {st }}$ and $U_{x}$ are the voltages in the reference and measuring cells and const ${ }_{2}$ is a cell constant similar to const 1 in Eq. (14).

The voltage fed onto the reference cell is typically fixed, and the adjustment for the birefringence compensation is carried out by changing the voltage of the measuring cell. The voltages of the reference and measuring cells were determined with the converter V3-23. Benzene was used in the reference cell of the birefringence compensation experiments. The Kerr constant $B$ of benzene at $\lambda=632.8 \mathrm{~nm}$ and $T=298 \mathrm{~K}$ equals to $3.54 \times 10^{-15} \mathrm{~m} \mathrm{~V}^{-2}$ [39].

The Kerr constants were also measured in reference to a standard phase-shifting plate using a phase detector in the recording amplifier. This type of measurements avoids the compensation procedure and gives the absolute values of the Kerr constant directly.

The error analysis for the measurements carried out with the above setups showed that for liquids with resistance $10^{9} \Omega \mathrm{cm}$ or above the accuracy of the compensation scheme was better than $0.1 \%$, with the errors arising primarily due 
to the inaccuracy in the measured voltages of the two cells. Other instrumental errors were small relative to the voltage error. The instrumental error of the absolute measurements was estimated at $0.4 \%$.

The refractive index of the liquids was measured with the Pulfrich IRF-23 refractometer. The relative error of the refractive index measurements equaled $0.02 \%$.

\subsection{Substance preparation}

It was essential for the birefringence measurements that the substances under study were purified, and kept dry and clean. Particular attention was given to the removal of impurities that could interfere with normal measurements. The necessary conditions for the electro-optical measurements included:

1) complete absence of dust in the liquid under study;

2) sufficient transparency of the liquid at the given wavelength;

3) complete absence of water in the liquid.

The cleaning and dehydration of the alkanes was carried out using the procedures described in Ref. [40].

\section{Results and discussion}

Table I shows the results of the EOKE measurements and refractive index of the series of $n$-alkanes under investigation. Confirming the literature data [42] obtained at $\lambda=546 \mathrm{~nm}$, the data of Table I show that the Kerr constant $B_{\exp }$ grows with increasing number of carbon atoms $\left(n_{\mathrm{C}}\right)$ in the alkane molecule. The smallest deviations between the experimental and theoretical values of the Kerr constant $\left(\Delta B=B_{\exp }-B_{i \text { calc }}\right)$ are seen with $B_{\mathrm{Z} 1}$ of Eq. (6), while the largest deviations occur with $B_{\mathrm{Z} 2}$ of Eq. (7). The $B_{\mathrm{S}}$ values calculated with Eq. (2) show the slowest increase in the Kerr constant with increasing $n_{\mathrm{C}}$ (Fig. 2).

The observed $\Delta B_{i}=f\left(n_{\mathrm{C}}\right)$ dependence fully corresponds to the increase in the IMI energy between $n$-alkane molecules in liquids with increasing $n_{\mathrm{C}}$ (Table II). Since $n$-alkanes carry no dipole moments $(\mu=0)$, the dispersive interaction $\left(E_{\text {disp }}^{0}\right)$ is the key contribution to the IMI energy. The dispersive interaction energy can be computed according to [45]:

$$
E_{\text {disp }}^{0}=\frac{1}{(4 \pi \varepsilon)^{2}} \frac{3 I_{i} \alpha_{i}^{2}}{4 R_{i i}^{6}}
$$

where $I_{i}$ is ionization potential, $a_{i}$ is average molecular polarizability, and $R_{i i}$ is average distance between the interacting $i$ molecules.

The distance between a pair of interacting molecules in a liquid $n$-alkane $\left(R_{i i}\right)$ can be found from the molecular volume $\left(V_{i}\right)$. If $r_{i i}$ is the radius of the cavity occupied by a single molecule in the liquid, the magnitude of $R_{i i}$ is twice the radius $R_{i i}=2 r_{i i}$. The X-ray studies of liquid $n$-alkanes [18] indicate that the average distance between the molecules in the first coordination sphere equals $5.38 \AA$. This value was used in the calculation of $E_{\text {disp }}^{0}$ reported in Table II. 
The Role of Intermolecular Interactions ...

\begin{tabular}{|c|c|c|}
\hline \\
\hline \multirow{12}{*}{ 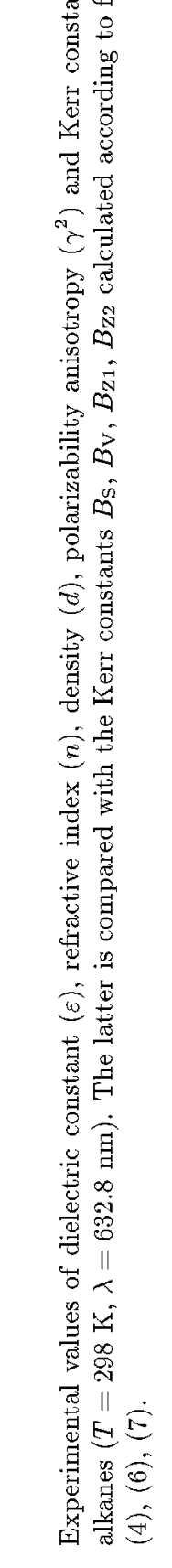 } & 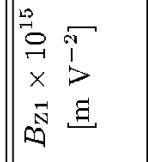 & 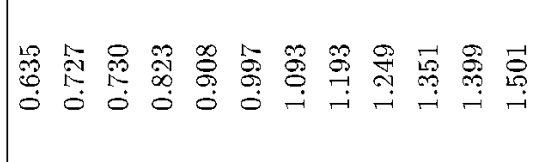 \\
\hline & 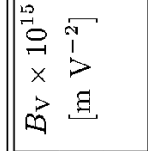 & 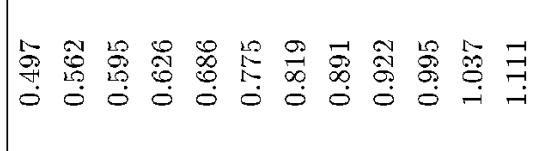 \\
\hline & 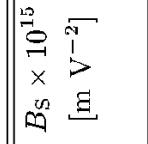 & 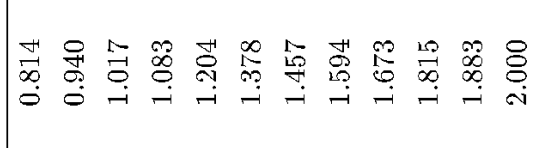 \\
\hline & 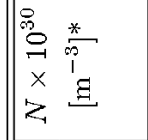 & 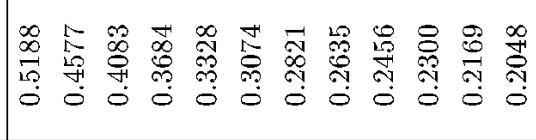 \\
\hline & 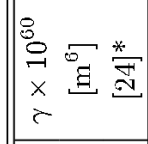 & 占 导 \\
\hline & 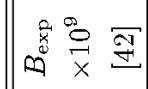 & 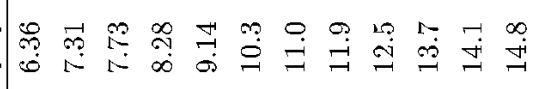 \\
\hline & 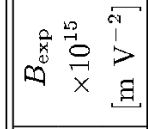 & | \\
\hline & 8 & 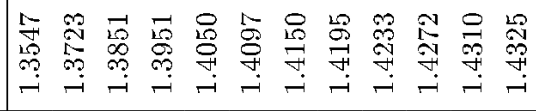 \\
\hline & 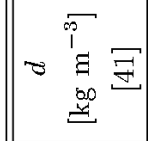 & 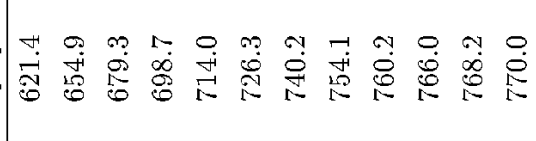 \\
\hline & $\omega \bar{\exists}$ & 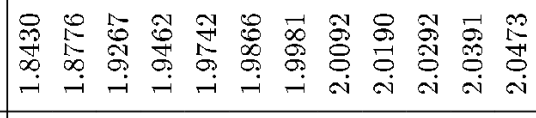 \\
\hline & 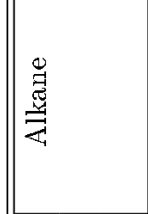 & 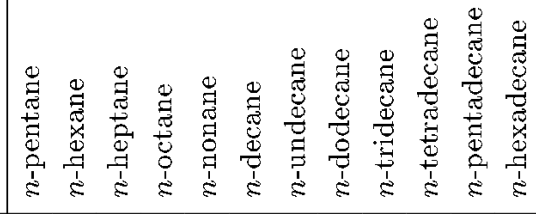 \\
\hline & 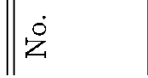 & $-\infty$ \\
\hline
\end{tabular}




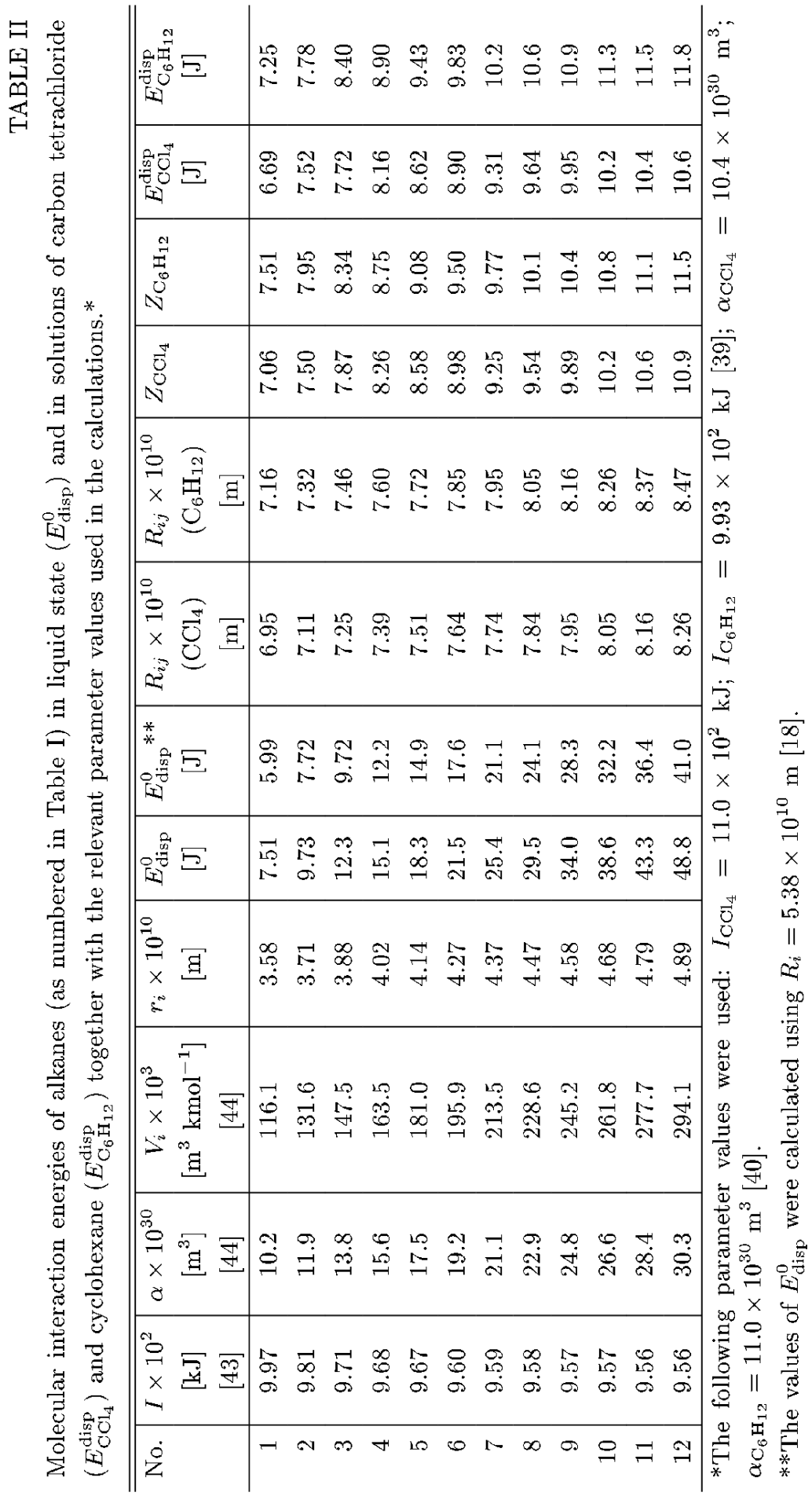




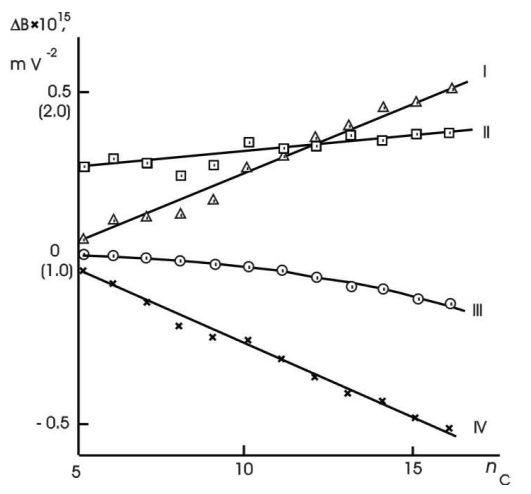

Fig. 2. Dependence of the difference between the experimental and calculated values of the Kerr constant $\Delta B=B_{\exp }-B_{i \text { calc }}$ on the number of carbon atoms $\left(n_{\mathrm{C}}\right)$ in $n$-alkane: I - for $\Delta B_{\mathrm{Z} 2}=B_{\exp }-B_{\mathrm{Z} 2}, \mathrm{II}-$ for $\Delta B_{\mathrm{S}}=B_{\exp }-B_{\mathrm{S}}$, III - for $\Delta B_{\mathrm{Z} 1}=$ $B_{\exp }-B_{\mathrm{Z} 1}, \mathrm{IV}-$ for $\Delta B_{\mathrm{V}}=B \exp -B_{\mathrm{V}} . B_{i \text { calc }}\left(B_{\mathrm{Z} 2}, B_{\mathrm{S}}, B_{\mathrm{Z} 1}, B_{\mathrm{V}}\right)$ are computed with Eqs. (2), (4), (6), (7). The scale of $\Delta B_{\mathrm{Z} 2}$ is given in parentheses.

The magnitudes of the molar Kerr constant ${ }_{m} K$ of the alkanes under investigation are given in Table III. The functional relationship between the molar Kerr constant and the number of atoms in the alkane molecule is illustrated in Fig. 3. Both ${ }_{m} K$ and $B$ grow with $n_{\mathrm{C}}$. The growth is especially fast in gas phase, where the interaction between the molecules is minimal. In the ideal case, when association or other kinds of IMI that affect molecular orientation are absent, the molar Kerr constant should not change from gas to liquid or solution. If an IMI of any form influences molecular orientation in a liquid, the ${ }_{m} K$ magnitude will change. This effect is clearly seen with the $n$-alkanes. IMI that may be present in the gas phase are greatly amplified in the liquid phase and solution.

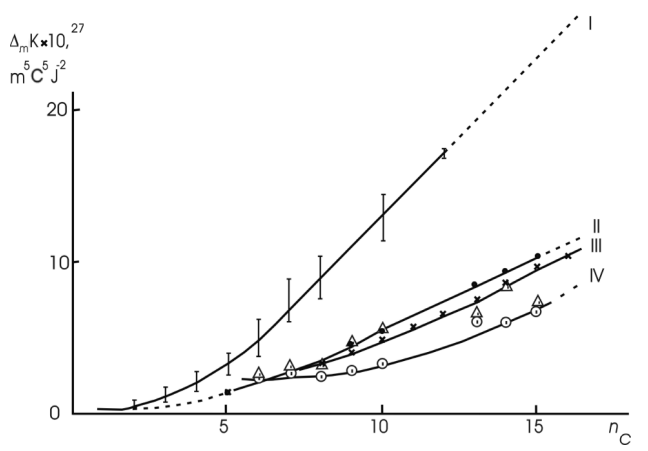

Fig. 3. Dependence of the molar Kerr constant $\left({ }_{m} K\right)$ of $n$-alkanes on the number of carbon atoms $\left(n_{\mathrm{C}}\right)$ in the molecule: I - in gas phase $\left({ }_{m} K_{\text {gas }}\right)$; II - in liquid phase $\left({ }_{m} K_{\text {liq }}\right)$; III — in liquid phase calculated using the data of this work and the Le Fevre equation (9); IV — in cyclohexane; $\triangle$ indicate the measurements in $\mathrm{CCl}_{4}$. The experimental ${ }_{m} K$ values are taken from Ref. [38]. 


\begin{tabular}{|c|c|c|}
\hline 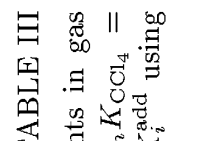 & 象 & 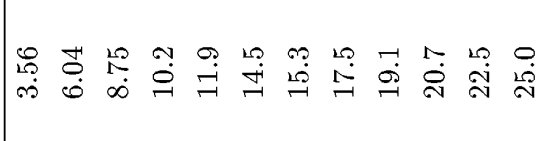 \\
\hline$\widehat{g}$ & 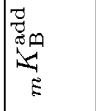 & 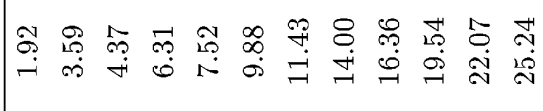 \\
\hline & 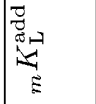 & 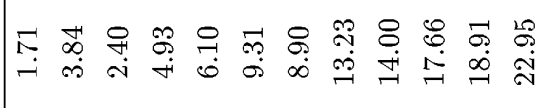 \\
\hline$\frac{\pi}{0} 0$ & $\underset{\Xi}{0}$ &  \\
\hline & $\underset{\Sigma}{\geq}$ & 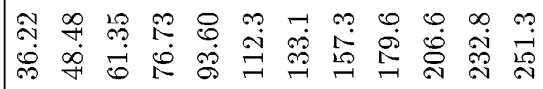 \\
\hline & 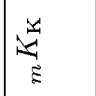 & 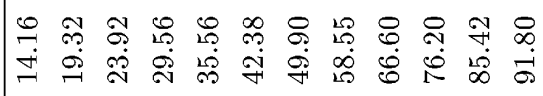 \\
\hline 5 & $\underbrace{\infty}_{\Sigma}$ & 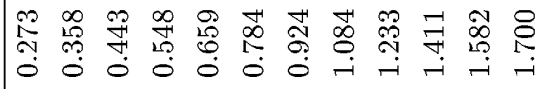 \\
\hline & 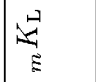 &  \\
\hline i &  & 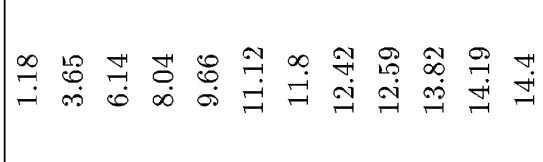 \\
\hline ì & 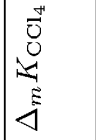 & 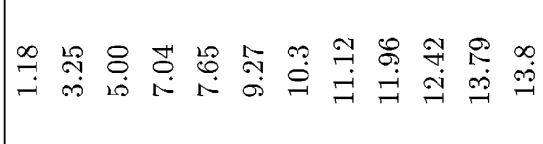 \\
\hline  & 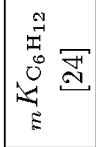 & 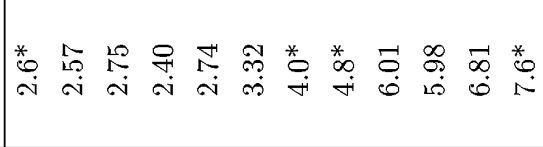 \\
\hline 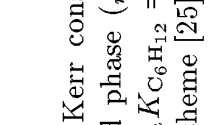 & 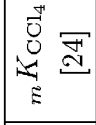 &  \\
\hline हేँ & 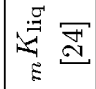 & 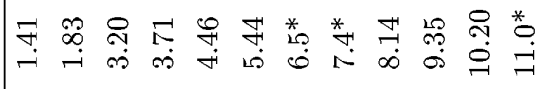 \\
\hline$\dot{0}$ & 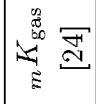 &  \\
\hline & $\dot{8}$ &  \\
\hline
\end{tabular}


The analysis of the solvent-solute interactions is performed in present according to the classification [46]:

$$
E=E_{i}+E_{i j}+E_{j j},
$$

where $i$ and $j$ mark solute and solvent, respectively.

Discrete-continuum model [47]. The fact that solute molecules in a dilute solution are surrounded by solvent molecules only, Fig. 4, allows for the following treatment of IMI. Molecular interactions in the first coordination shell are computed explicitly, and the energy of interaction between the solute molecules is neglected.

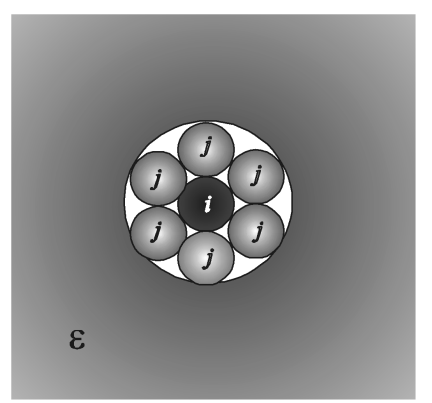

Fig. 4. The discrete-continuum model of interaction of a solute molecule $(i)$ with solvent molecules $(j)$ in a medium with dielectric permittivity $\varepsilon$.

The solute-solvent interaction within the first coordination shell can be expressed as [47]:

$$
\begin{aligned}
E= & \frac{1}{(4 \pi \varepsilon)^{2}}\left(\frac{Z}{R^{6}}\right)_{1}\left[\varphi_{\mathrm{disp}} \frac{3}{2} \frac{I_{i} I_{j}}{I_{i}+I_{j}} \alpha_{i} \alpha_{j}\right. \\
& \left.+\varphi_{\mathrm{d}-\mathrm{d}} \frac{2}{3} \frac{\mu_{i}^{2} \mu_{j}^{2}}{k T}+\varphi_{\mathrm{d}-i \mathrm{~d}}\left(\mu_{i}^{2} \alpha_{j}+\mu_{j}^{2} \alpha_{i}\right)\right],
\end{aligned}
$$

where $Z$ is the number of solvent molecules in the first coordination shell. Since interactions in condensed phases include many particles, and Eq. (18) represents only the pairwise component of the interaction, the corrections $\varphi_{i}$ for many-body effects are introduced. The correction is assumed constant for a series of solutes in the same solvent. In the case of non-polar molecules such as alkanes in non-polar solvents such as $\mathrm{CCl}_{4}$ and $\mathrm{C}_{6} \mathrm{H}_{12}$ Eq. (18) simplifies to

$$
E_{i j}^{\text {disp }}=\frac{1}{(4 \pi \varepsilon)^{2}}\left(\frac{Z}{R^{6}}\right)_{1} \frac{3}{2} \frac{I_{i} I_{j}}{I_{i}+I_{j}} \alpha_{i} \alpha_{j} .
$$

Equation (19) was used to analyze the IMI energy of the $n$-alkane solutes with the $\mathrm{CCl}_{4}$ and $\mathrm{C}_{6} \mathrm{H}_{12}$ solvents. The results are presented in Table II. As expected, the data indicate that the IMI energy grows with the size of the molecule $n_{\mathrm{C}}$. The 
difference between the gas phase and solution values of the molar Kerr constant $\Delta_{m} K={ }_{m} K_{\text {gas }}-{ }_{m} K_{\text {sol }}$ increases with the IMI energy (Fig. 5). The correlation between $\Delta_{m} K$ and the strength of the IMI supports the conclusion that the IMI plays the main role in the reduction of ${ }_{m} K$ of the alkanes during the transition from gas into solution.

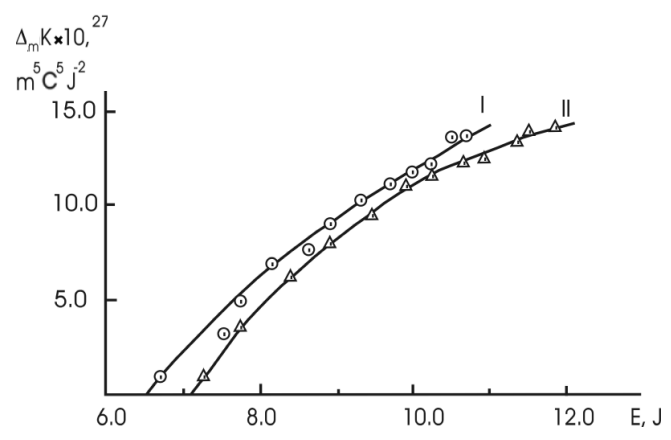

Fig. 5. Dependence of $\Delta_{m} K={ }_{m} K_{\text {gas }}-{ }_{m} K_{\text {sol }}$ on the energy of dispersion interaction of $n$-alkanes with the solvent molecules: $\mathrm{I}-$ in $\mathrm{CCl}_{4}$, II - in $\mathrm{C}_{6} \mathrm{H}_{12}$.

The models of the EOKE producing Eqs. (8)-(13) for the molar Kerr constant were used to calculate the ${ }_{m} K$ values on the basis of the experimental data of Table I. As follows from the ${ }_{m} K_{i}$ values presented in Table III, as well as from the magnitudes of $B_{i}$ shown in Table II, the Kerr constants in the condensed phase differ strongly from the corresponding gas phase values ${ }_{m} K_{\text {gas }}$. The greatest deviations are observed for ${ }_{m} K_{\mathrm{V}}$ calculated according to Eq. (10), while the smallest deviations are seen with ${ }_{m} K_{\mathrm{L}}$ computed with Eq. (9). Since the dispersive interaction energy $E_{\text {disp }}$ correlates with the $n$-alkane length $n_{\mathrm{C}}$, the $\left|\Delta_{m} K_{i}\right|$ magnitudes increase steadily (Fig. 6), but with different slopes depending on the internal field model.

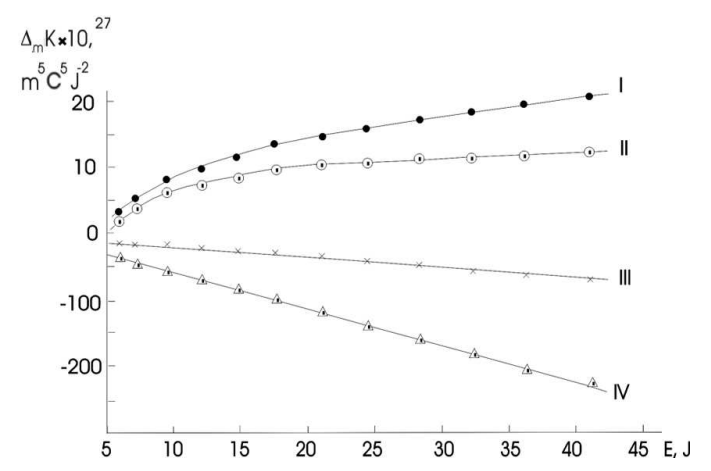

Fig. 6. The relationship between $\Delta_{m} K_{i}\left(\Delta_{m} K_{i}={ }_{m} K_{\text {gas }}-{ }_{m} K_{\text {liq }}\right)$ and the energy $(E)$ of dispersion interaction between alkane molecules in liquid phase: I $\Delta_{m} K_{\mathrm{B}}$, Eq. (11); II $-\Delta_{m} K_{\mathrm{L}}$, Eq. (9); III $-\Delta_{m} K_{\mathrm{K}}$, Eq. (8); IV $-\Delta_{m} K_{\mathrm{V}}$, Eq. (10). 
Liquids possess local order $[48,49]$ as evidenced by X-ray diffraction in particular $[17,18,50,51]$, revealing liquid local order that closely resembles the crystal structure. For example, $n$-pentane shows both a parallel local order, where the molecules are arranged in the same direction and are shifted along the molecular axis by about $2.5 \AA$ with respect to each other, and a perpendicular local structure resembling letter $\mathrm{T}$ [18]. In addition to the local order, and in contrast to crystals, molecules in liquid participate in collective rotations [52].

The ${ }_{m} K$ values are very sensitive to the changes of the molecular and supermolecular structures in liquids. A number of tensor-additive schemes for the computation of the ${ }_{m} K$ magnitudes from polarizabilities of individual bonds have been developed [25]. Such tensor-additive values of ${ }_{m} K_{i}^{\text {add }}$ were computed (Table III) based on the data of Ref. [17] showing that $n$-alkanes prefer the periplanar conformation $(a p)$ (Fig. 7). The calculations were performed using the tensor-additive schemes of Le Fevre [53], Caristan et al. [54] and Vuks [55]. The Le Fevre approximation is most popular and assumes that the $\mathrm{C}-\mathrm{H}$ bond is isotropic $\left(b_{\mathrm{L}}=b_{\mathrm{T}}=b_{\mathrm{V}}\right)$. The Le Fevre scheme uses the following values for the half-axis of the bond and group polarizability ellipsoids given in $\AA^{3}$ [53]. C-C: $b_{\mathrm{L}}=0.97$, $b_{\mathrm{T}}=b_{\mathrm{V}}=0.26 ; \mathrm{C}-\mathrm{CH}_{3}: b_{\mathrm{L}}=3.00, b_{\mathrm{T}}=b_{\mathrm{V}}=2.18 ; \mathrm{C}-\mathrm{H}: b_{\mathrm{L}}=b_{\mathrm{T}}=b_{\mathrm{V}}=0.63$. The corresponding values used in the Caristan scheme [54] are $\mathrm{C}-\mathrm{C}: b_{\mathrm{L}}=1.38$, $b_{\mathrm{T}}=b_{\mathrm{V}}=0.07 ; \quad \mathrm{C}-\mathrm{CH}_{3}: b_{\mathrm{L}}=3.32, b_{\mathrm{T}}=b_{\mathrm{V}}=2.10 ; \mathrm{C}-\mathrm{H}: b_{\mathrm{L}}=0.81$, $b_{\mathrm{T}}=b_{\mathrm{V}}=0.59$. The work of Vuks [55] made systematic assumptions about the anisotropies of the $\mathrm{C}-\mathrm{H}$ and $\mathrm{C}-\mathrm{C}$ bonds that were further tested experimentally. As the first step in the determination of the bond polarizability parameters, the range of possible absolute polarizability values was established. Then, the bond polarizability parameters were derived based on the experimental data for ethane and related molecules, and under the assumption that in a homological series the parameters of the $\mathrm{C}-\mathrm{H}$ bond do not change. The procedure resulted in the following values of the bond polarizability anisotropy: $b_{\mathrm{L}}(\mathrm{C}-\mathrm{C})=1.53$, $b_{\mathrm{T}}(\mathrm{C}-\mathrm{C})=b_{\mathrm{V}}(\mathrm{C}-\mathrm{C})=0.16 ; b_{\mathrm{L}}(\mathrm{C}-\mathrm{H})=0.81, b_{\mathrm{T}}(\mathrm{C}-\mathrm{H})=b_{\mathrm{V}}(\mathrm{C}-\mathrm{H})=0.57$ [55]. The molar Kerr constants obtained by the tensor-additive schemes due to Le Fevre ${ }_{m} K_{\mathrm{L}}^{\text {add }}$, Caristan ${ }_{m} K_{\mathrm{B}}^{\text {add }}$ and Vuks ${ }_{m} K_{\mathrm{V}}^{\text {add }}$ are presented in Table III. The com-
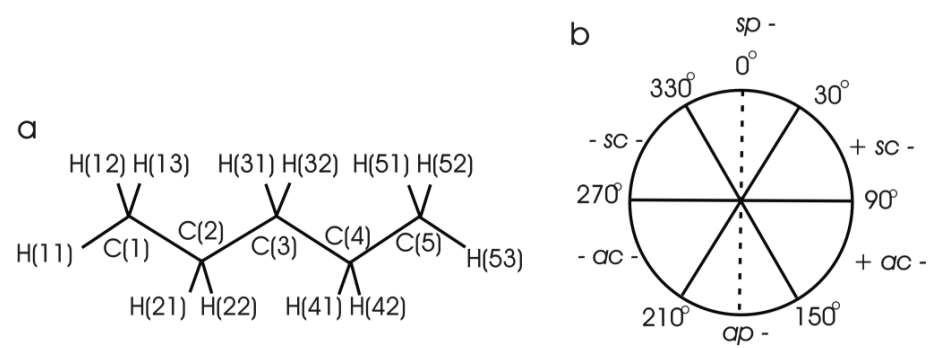

Fig. 7. The conformation of $n$-pentane molecule (a) and the designations of the conformation types (b): $s c$ - synclinal, $a c$ - anticlinal, $s p$ - synclinal-antiperiplanar, ap - antiperiplanar. 
parison of the calculated ${ }_{m} K_{i}^{\text {add }}$ and experimental ${ }_{m} K_{\text {gas }}$ values shows that the additive scheme of Vuks shows the best agreement with the experiment.

The molar Kerr constants calculated using the tensor-additive schemes described in the previous paragraph were computed for the most stable conformation of $n$-alkanes. Generally, however, alkanes have significant conformational flexibility and may exhibit a set of conformers rather than a single fixed configuration. In order to estimate the conformational flexibility of $n$-alkanes as well as the fraction of each conformer that exists in thermodynamic equilibrium at a given temperature, it is necessary to consider the potential energy $(U)$ of the molecules as a function of the internal angles [56]. The torsion angle dependence of the potential energy $U$ of ethane-like molecules is shown in Fig. 8. The energy difference between the maximum and minimum defines the height of the torsion energy barrier that must be overcome during the transition between the conformers. Only the first several members $\left(\mathrm{C}_{2}-\mathrm{C}_{4}\right)$ of the $n$-alkane series exhibit very good agreement between the calculated ${ }_{m} K_{\mathrm{V}}^{\text {add }}$ and experimental ${ }_{m} K_{\text {gas }}$ values: for $\mathrm{C}_{2} \mathrm{H}_{6} 1.021$ (1.0); $\mathrm{C}_{3} \mathrm{H}_{8} 1.535$ (1.5); $\mathrm{C}_{4} \mathrm{H}_{10} 2.552$ (2.6), correspondingly. The longer homologues in the series show a larger divergence between ${ }_{m} K_{i}^{\text {add }}$ and ${ }_{m} K_{\text {gas }}$ (Table III) because of the conformation flexibility that increases with $n_{\mathrm{C}}$. In particular, the longer $n$-alkanes show appreciable amounts of both synclinal-antiperiplanar $(s p)$ and doubly antiperiplanar (ap) conformations [57].

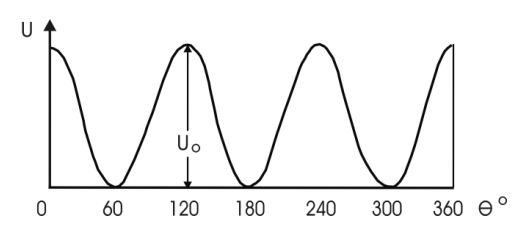

Fig. 8. Dependence of potential energy $(U)$ on the internal rotation angle $\left(\theta^{\circ}\right)$ for ethane-like molecules.

Due to the high conformational flexibility, it is not straightforward to quantify the effect of local structures on the EOKE values in liquid $n$-alkanes. For instance, the experimental molar Kerr constant of liquid $n$-pentane equals ${ }_{m} K_{\exp }=1.41 \times 10^{-27}$. The theoretical value for the monomer calculated with the tensor-additive scheme of Vuks [55] is a factor of two larger ${ }_{m} K_{\mathrm{V}}^{\mathrm{add}}=3.56 \times 10^{-27}$. The corresponding calculations for the two dimers that dominate the local order of liquid $n$-pentane [17] give ${ }_{m} K_{\mathrm{T}}^{\text {calc }}=0,{ }_{m} K_{\|}^{\text {calc }}=1.10 \times 10^{-27}$. These results testify that indeed the effect of the local order on the EOKE in the liquid is strong, and that liquid $n$-alkanes exist in equilibrium of the T-shaped dimers and other forms having ${ }_{m} K>0$.

The study of the local structures and their role in EOKE is greatly facilitated by the use of conformationally stable compounds with known liquid order, such as benzene, naphthalene, carbon disulfide, etc. Studies of EOKE in these liquids are planned for the near future. 


\section{Conclusions}

Simple theories describe EOKE qualitatively and give correct quantitative trends, but do not make reliable quantitative predictions for the magnitudes of the Kerr constant $B$ and molar Kerr constant ${ }_{m} K$ even for such simple liquids as $n$-alkanes. IMI between the molecules in the condensed phase greatly affects the values of the Kerr constants. The growth of the IMI energy reduces the magnitudes of $B$ and ${ }_{m} K$ in liquid $n$-alkanes relative to the gas phase values. All theories predict this effect regardless of the specific internal field model used for the description of birefringence in pure liquids and solutions. Comparison of the molar Kerr constants calculated according to the tensor-additive schemes with the measured values showed that good agreement between theory and experiment can be reached only if the theory accounts for the local liquid order. The collective molecular orientations greatly influence the liquid response to the electric field and determine the mechanism of EOKE in the condensed phase.

\section{References}

[1] S. Kielich, Acta Phys. Pol. 22, 299 (1962).

[2] S. Kielich, Molecular Nonlinear Optics, PWN, Warszawa-Poznań 1977 (in Polish).

[3] V.V. Prezhdo, M.V. Khashchina, V.A. Zamkov, Electro-optical Investigation in Physics and Chemistry, Vyshcha Shkola, Kharkov 1982, p. 152 (in Russian).

[4] D. Dymaczewska, M. Surma, Acta Phys. Pol. A 62, 105 (1982).

[5] Z. Błaszczak, Acta Phys. Pol. A 71, 601 (1987).

[6] Z. Błaszczak, Acta Phys. Pol. A 69, 621 (1986).

[7] J.J. Stankus, M.M. Tazazma, M.D. Fayer, J. Phys. Chem. 98, 313 (1994).

[8] M. Born, Ann. Phys. 55, 177 (1918).

[9] R. Patz, M.T. Ratzsch, Z. Phys. Chem. 265, 724 (1984).

[10] W. Pyzuk, H. Majgier-Baranowska, J. Ziolo, J. Chem. Phys. 59, 111 (1981).

[11] O.V. Prezhdo, S.A. Tyurin, M. Pisarczyk, V.V. Prezhdo, Acta Phys. Pol. A 101, $477(2002)$.

[12] M.F. Vuks, Zh. Fiz. Khim. 52, 134 (1978).

[13] A.D. Buckingham, Trans. Faraday Soc. 77, 577 (1981).

[14] M.F. Vuks, Electrical and Optical Properties of Molecules and Condensed Medium, Leningrad Univ., Leningrad 1984 (in Russian).

[15] J.M. Neto, A.B. Villaverde, J. Phys., Condens. Matter 8, 2791 (1996).

[16] R. Patz, T.M. Ratzsch, Z. Phys. Chem. 260, 788 (1979).

[17] Z. Bochyński, H. Drozdowski, Phys. Chem. Liq. 39, 189 (2001).

[18] Z. Bochyński, H. Drozdowski, Acta Phys. Pol. A 88, 1089 (1995).

[19] H.A. Stuart, Die Struktur des freien Molekuls, Springer, Berlin 1952.

[20] V.A. Zamkov, Opt. Spektrosk. (USSR) 15, 654 (1963). 
[21] V.A. Zamkov, Ukrain. Fiz. Zh. 7, 720 (1962).

[22] V.A. Zamkov, in: Optical Investigation in Liquids and Solutions, Ed. T. Akhadov, Fan, Tashkent 1965, p. 128 (in Russian).

[23] G. Briegleb, Z. Phys. Chem. B 14, 97 (1931).

[24] A.N. Vereshchagin, Characteristics of Molecular Anisotropy of Polarizability, Nauka, Moscow 1982 (in Russian).

[25] A.N. Vereshchagin, Polarizability of Molecules, Nauka, Moscow 1980 (in Russian).

[26] C.G. Le Fevre, R.J.W. Le Fevre, Rev. Pure Appl. Chem. 5, 261 (1955).

[27] L. Onsager, J. Am. Chem. Soc. 58, 486 (1936).

[28] S. Kielich, Acta Phys. Pol. 34, 1093 (1968).

[29] A.D. Buckingham, P.J. Stiles, G.L.D. Ritchie, Trans. Faraday Soc. 67, 577 (1971).

[30] A.D. Buckingham, J.A. Pople, Proc. Phys. Soc. A 68, 905 (1955).

[31] A. Proutiere, Contribution a l'etude de la birefringence electrique des liquids, Univ. Bordeaux, Bordeaux 1973, p. 164.

[32] B. Ladanyi, T. Keyes, Mol. Phys. 34, 1693 (1977); 39, 645 (1980).

[33] B. Ladanyi, T. Keyes, Canad. J. Phys. 59, 1421 (1981).

[34] S.B. Bulgarevich, D.Ya. Movshovich, Z. Obshch. Khim. 55, 1690 (1985).

[35] S.B. Bulgarevich, D.Ya. Movshovich, M.G. Grunfest, Z. Obshch. Khim. 59, 913 (1989).

[36] A.N. Narten, J. Chem. Phys. 67, 2102 (1977).

[37] M.V. Khashchina, V.S. Raff, V.A. Zamkov, Instr. Exp. Techn. 18, 200 (1975).

[38] J.J. Stankus, M. Terazma, M.D. Fayer, J. Phys. Chem. 98, 313 (1994).

[39] S.A. Myers, E.J. Robinson, J. Chem. Phys. 58, 1356 (1973).

[40] D.D. Perrin, D.R. Perrin, W.L.F. Armarego, Purification of Laboratory Chemicals, Pergamon, Oxford 1980; Recommend Methods for the Purification of Solvents and Tests for Impurities, Pergamon, Oxford 1982.

[41] Solvents Database, Ed. G. Wypych, Chemtech Publishing, Toronto 2000.

[42] A. Proutiere, J.G.R. Baudet, M. Camail, J. Chim. Phys. 71, 1439 (1974).

[43] Energy of Chemical Bonds Breaking. Ionization Potentials and Electron Affinity, Ed. V.N. Kondratiev, Nauka, Moscow 1974 (in Russian).

[44] Y. Markus, The Properties of Solvents. Wiley Series in Solution Chemistry, Vol. 4, Wiley, Chichester 1999.

[45] E. Silla, A. Arnau, J. Tunon, in: Handbook of Solvents, Ed. G. Wypych, ChemTech Publishing, Toronto 2001, p. 13.

[46] Ch. Reichardt, Solvents and Solvent Effects in Organic Chemistry, Wiley-VCH, Weinheim 2003.

[47] V.V. Prezhdo, M. Jagiełło, I.I. Melnik, M.V. Prezhdo, Separation Sci. Tech. 37, 2875 (2002).

[48] J.P. Hansen, I.R. Mc Donald, Theory of Simple Liquids, Academic Press, London 1986.

[49] A.Z. Patashinski, A.C. Mitus, M.A. Ratner, Phys. Rep. 288, 409 (1997). 
[50] T. Jijima, K. Nishikawa, J. Mol. Struct. 352/353, 213 (1995).

[51] Z. Bochyński, L. Dejneka, H. Drozdowski, J. Mol. Struct. 524, 227 (2000).

[52] D. Shelton, Chem. Phys. Lett. 325, 513 (2000).

[53] R.J.W. Le Fevre, Adv. Phys. Org. Chem. 3, 1 (1965).

[54] A. Caristan, P. Bothorel, H. Bodot, J. Chim. Phys. 66, 1009 (1969).

[55] M.F. Vuks, Light Scattering in Gas, Liquids $\&$ Solutions, Leningrad Univ., Leningrad 1977 (in Russian).

[56] Conformational Analysis of Hydrocarbons $\&$ Their Derivatives, Ed. B.A. Arbuzov, Nauka, Moscow 1990 (in Russian).

[57] R.A. Bonhem, L.S. Bartell, D. Kohl, J. Am. Chem. Soc. 81, 4765 (1959). 